\title{
Pick Interpolation for free holomorphic functions
}

\author{
Jim Agler * \\ U.C. San Diego \\ La Jolla, CA 92093
}

\author{
John E. McCarthy ${ }^{\dagger}$ \\ Washington University \\ St. Louis, MO 63130
}

September 1, 2021

\section{Introduction}

A holomorphic function in $d$ (scalar) variables behaves locally like a polynomial. Given such a function $\phi$, one can evaluate it also on $d$-tuples of commuting matrices whose joint spectrum lies in the domain of $\phi$. A free holomorphic function is a generalization of this notion, where the matrices are no longer required to commute. We replace polynomials by free polynomials, i.e. polynomials in non-commuting variables, and consider functions that are locally limits of free polynomials.

To make this precise, let us first define $\mathbb{M}_{n}^{d}$ to be the set of all $d$-tuples of $n$-by- $n$ complex matrices, and $\mathbb{M}^{[d]}=\cup_{n=1}^{\infty} \mathbb{M}_{n}^{d}$. Let $\mathbb{P}^{d}$ denote the algebra of free polynomials in $d$ variables. A graded function defined on a subset of $\mathbb{M}^{[d]}$ is a function $\phi$ with the property that if $x \in \mathbb{M}_{n}^{d}$, then $\phi(x) \in \mathbb{M}_{n}$.

Definition 1.1. An nc-function is a graded function $\phi$ defined on a set $D \subseteq \mathbb{M}^{[d]}$ such that

i) If $x, y, x \oplus y \in D$, then $\phi(x \oplus y)=\phi(x) \oplus \phi(y)$.

ii) If $s \in \mathbb{M}_{n}$ is invertible and $x, s^{-1} x s \in D \cap \mathbb{M}_{n}^{d}$ then $\phi\left(s^{-1} x s\right)=$ $s^{-1} \phi(x) s$.

\footnotetext{
*Partially supported by National Science Foundation Grant DMS 1068830

${ }^{\dagger}$ Partially supported by National Science Foundation Grant DMS DMS 1300280
} 
Nc-functions have been studied for a variety of reasons: by Taylor [25], in the context of the functional calculus for non-commuting operators; Voiculescu $[26,27]$, in the context of free probability; Popescu $[19,20,21,22]$, in the context of extending classical function theory to $d$-tuples of bounded operators; Ball, Groenewald and Malakorn [7], in the context of extending realization formulas from functions of commuting operators to functions of non-commuting operators; Alpay and Kalyuzhnyi-Verbovetzkii [4] in the context of realization formulas for rational functions that are $J$-unitary on the boundary of the domain; Helton [10] in proving positive matrix-valued functions are sums of squares; and Helton, Klep and McCullough [11, 12] and Helton and McCullough [13] in the context of developing a descriptive theory of the domains on which LMI and semi-definite programming apply. Recently, Kaliuzhnyi-Verbovetskyi and Vinnikov have written a monograph [16] that gives a panoramic view of the developments in the field to date, and establishes their Taylor-Taylor formula for nc-functions.

There are two topologies that we wish to consider on $\mathbb{M}^{[d]}$. The first is called the disjoint union topology: a set $U$ is open in the disjoint union topology if and only if $U \cap \mathbb{M}_{n}^{d}$ is open for every $n$. This topology is too fine for some purposes; for example, compact sets must have a bound on the size of the matrices that they contain. The other topology we wish to consider is the free topology, which is most conveniently defined by giving a basis. A basic free open set in $\mathbb{M}^{[d]}$ is a set of the form

$$
G_{\delta}=\left\{x \in \mathbb{M}^{[d]}:\|\delta(x)\|<1\right\}
$$

where $\delta$ is a $J$-by- $J$ matrix with entries in $\mathbb{P}^{d}$. We define the free topology to be the topology on $\mathbb{M}^{[d]}$ which has as a basis all the sets $G_{\delta}$, as $J$ ranges over the positive integers, and the entries of $\delta$ range over all polynomials in $\mathbb{P}^{d}$. (Notice that $G_{\delta_{1}} \cap G_{\delta_{2}}=G_{\delta_{1} \oplus \delta_{2}}$, so these sets do form the basis of a topology). The free topology is a natural topology when considering semi-algebraic sets.

Definition 1.2. A free holomorphic function $\phi$ on a free open set $U$ in $\mathbb{M}^{[d]}$ is a function that is locally a bounded nc-function, i.e. for every $x$ in $U$ there is a basic free open set $G_{\delta} \subseteq U$ that contains $x$ and such that $\left.\phi\right|_{G_{\delta}}$ is a bounded nc-function.

It is a principal result of [1] that free holomorphic functions are locally approximable by free polynomials (see Theorem 2.1 below). 
The main result of this note is a criterion for solving a Pick interpolation problem on a basic open set, Theorem 1.3 below, and its generalization to extending bounded free holomorphic functions off free varieties, Theorem 1.5.

Let $H^{\infty}(U)$ denote the bounded free holomorphic functions on a free open set $U$ with the supremum norm, and let $H_{1}^{\infty}(U)$ denote the closed unit ball of $H^{\infty}(U)$. For $1 \leq i \leq N$, let $\lambda_{i} \in G_{\delta} \cap \mathbb{M}_{n_{i}}^{d}$ and let $w_{i} \in \mathbb{M}_{n_{i}}$. The Pick problem is to determine whether or not there is a function in $H_{1}^{\infty}(U)$ that maps each $\lambda_{i}$ to the corresponding $w_{i}$.

Note first that if $U$ is closed under direct sums, then by letting $\Lambda=\oplus_{i=1}^{N} \lambda_{i}$ and $W=\oplus_{i=1}^{N} w_{i}$, the original $N$ point problem is the same as solving the one point Pick problem of mapping $\Lambda$ to $W$. Secondly, unlike in the scalar case, one cannot always solve the Pick problem if one drops the norm constraint. For example, no holomorphic function maps

$$
\left(\begin{array}{ll}
0 & 1 \\
0 & 0
\end{array}\right) \quad \text { to } \quad\left(\begin{array}{ll}
1 & 0 \\
0 & 0
\end{array}\right) \text {. }
$$

To state the theorem, let us make the following definitions for $\Lambda$ in $\mathbb{M}^{[d]}$. Define

$$
\mathcal{I}_{\Lambda}=\left\{p \in \mathbb{P}^{d}: p(\Lambda)=0\right\}
$$

and

$$
V_{\Lambda}=\left\{x \in \mathbb{M}^{[d]}: p(x)=0 \text { whenever } p \in \mathcal{I}_{\Lambda}\right\}
$$

Let

$$
\mathbb{M}_{\Lambda}=\left\{p(\Lambda): p \in \mathbb{P}^{d}\right\}
$$

Note that since $\mathbb{M}_{\Lambda}$ is a finite dimensional vector space, it is closed.

Theorem 1.3. Let $\Lambda \in G_{\delta} \cap \mathbb{M}_{n}^{d}$ and $W \in \mathbb{M}_{n}$. There exists a function $\phi$ in the closed unit ball of $H^{\infty}\left(G_{\delta}\right)$ such that $\phi(\Lambda)=W$ if and only if

(i) $W \in \mathbb{M}_{\Lambda}$, so there exists $p_{0} \in \mathbb{P}^{d}$ such that $p_{0}(\Lambda)=W$.

(ii) $\sup \left\{\left\|p_{0}(x)\right\|: x \in V_{\Lambda} \cap G_{\delta}\right\} \leq 1$.

We prove this theorem in Section 3. Note that when $d=1$, the question of whether $p_{0}$ can be found satisfying $p_{0}(\Lambda)=W$ can be resolved by looking at the Jordan canonical form of $\Lambda$. In this basis, the algebra $\mathbb{M}_{\Lambda}$ has a straightforward description. When $d>1$, the determination of $\mathbb{M}_{\Lambda}$ is more delicate; generically ${ }^{1}$, however, the algebra $\mathbb{M}_{\Lambda}$ will be all of $\mathbb{M}_{n}$.

\footnotetext{
${ }^{1}$ For example, if $\Lambda^{1}$ has $n$ distinct eigenvalues and $\Lambda^{2}$ has no non-zero entry when the matrix is expressed in the basis given by the eigenvectors of $\Lambda^{1}$.
} 
In Section 4, we give a description in Theorem 4.6 of all the solutions of a (solvable) Pick problem - this is called the Nevanlinna problem. Our approach is indebtted to the solution in the scalar case by J. Ball, T. Trent and V. Vinnikov [6].

Theorem 1.3 has a remarkable corollary. Suppose $\mathfrak{A}$ is an algebra in $\mathbb{P}^{d}$, and let $\mathfrak{v}=\operatorname{Var}(\mathfrak{a})$ be given by

$$
\mathfrak{v}=\left\{x \in \mathbb{M}^{d}: p(x)=0 \forall p \in \mathfrak{A}\right\} .
$$

If $\Lambda$ is in $\mathfrak{v}$, then $\mathfrak{A} \subseteq \mathcal{I}_{\Lambda}$, and $V_{\Lambda} \subseteq \mathfrak{v}$. Let $U$ be a free open set in $\mathbb{M}^{[d]}$; we shall say that a function $f$ defined on $\mathfrak{D} \cap U$ is free holomorphic if, for every point $x$ in $\mathfrak{v} \cap U$ there is a basic free open set $G_{\delta} \subseteq U$ containing $x$ and a free holomorphic function $\psi$ defined on $G_{\delta}$ such that $\left.\psi\right|_{\mathfrak{v} \cap G_{\delta}}=\left.f\right|_{\mathfrak{v} \cap G_{\delta}}$.

In the scalar case, every holomorphic function defined on an analytic variety inside a domain of holomorphy extends to a holomorphic function on the whole domain, by a celebrated theorem of H. Cartan [8]. The geometric conditions that guarantee that all bounded holomorphic functions extend to be bounded on the whole domain have been investigated by Henkin and Polyakov [15] and Knese [17]; however, even when bounded extensions exist, the extension is almost never isometric [3]. But in the matrix case, any bounded free holomorphic function on $\mathfrak{v} \cap G_{\delta}$ does extend to a free holomorphic function on $G_{\delta}$ with the same norm.

Theorem 1.5. Let $\mathfrak{D}$ be as in (1.4), and let $\delta$ be a matrix of free polynomials such that $\mathfrak{D} \cap G_{\delta}$ is non-empty. Let $f$ be a bounded free holomorphic function defined on $G_{\delta} \cap \mathfrak{V}$. Then there is a free holomorphic function $\phi$ on $G_{\delta}$ that extends $f$ and such that

$$
\|\phi\|_{H^{\infty}\left(G_{\delta}\right)}=\sup _{x \in \mathfrak{v} \cap G_{\delta}}\|f(x)\|
$$

We prove this in Section 5. In Section 6 we give some applications.

The definition (1.4) naturally leads one to ask what the ideal of $\mathfrak{v}$, the set

$$
I_{\mathfrak{v}}=\left\{p \in \mathbb{P}^{d}: p(x)=0 \forall x \in \mathfrak{v}\right\},
$$

is. In the complex case, the answer is simpler than in the scalar case, at least if $\mathfrak{A}$ is finitely generated. In [14], Bergman, Helton and McCullough proved that $I_{\mathfrak{v}}$ is the smallest ideal containing $\mathfrak{A}$, provided this ideal is finitely generated. The real (self-adjoint) case is more subtle — see e.g. [9]. 


\section{Background material}

We shall need some results from [1]. The first we have already referenced:

Theorem 2.1. Let $D$ be a free domain and let $\phi$ be a graded function defined on $D$. Then $\phi$ is a free holomorphic function if and only if $\phi$ is locally approximable by polynomials.

The second, [1, Thm 8.1], says that a function is in $H_{1}^{\infty}\left(G_{\delta}\right)$ if and only if it has a free $\delta$-realization.

Definition 2.2. Let $\phi$ be a graded function on $G_{\delta}$, where $\delta$ is a $J$-by- $J$ matrix of free polynomials. A free $\delta$-realization of $\phi$ is a Hilbert space $\mathcal{L}$, an isometry $V: \mathbb{C} \oplus\left(\mathbb{C}^{J} \otimes \mathcal{L}\right) \rightarrow \mathbb{C} \oplus\left(\mathbb{C}^{J} \otimes \mathcal{L}\right)$ that can be written

$$
\begin{aligned}
& \mathbb{C} \quad \mathbb{C}^{J} \otimes \mathcal{L} \\
& V=\underset{\mathbb{C}^{J} \otimes \mathcal{L}}{\mathbb{C}}\left(\begin{array}{ll}
A & B \\
C & D
\end{array}\right),
\end{aligned}
$$

and such that

$$
\begin{aligned}
& \phi(x)=\operatorname{id}_{\mathbb{C}^{n}} \otimes A+ \\
& \quad\left(\operatorname{id}_{\mathbb{C}^{n}} \otimes B\right)\left(\delta(x) \otimes \operatorname{id}_{\mathcal{L}}\right)\left[\operatorname{id}_{\mathbb{C}^{n}} \otimes \operatorname{id}_{\mathbb{C}^{J} \otimes \mathcal{L}}-\left(\operatorname{id}_{\mathbb{C}^{n}} \otimes D\right)\left(\delta(x) \otimes \operatorname{id}_{\mathcal{L}}\right)\right]^{-1} \operatorname{id}_{\mathbb{C}^{n}} \otimes C
\end{aligned}
$$

for all $x \in G_{\delta} \cap \mathbb{M}_{n}^{d}$.

We call $\phi$ the transfer function of $V$ (where $\delta$ is understood).

Theorem 2.3. Let $\phi$ be a graded function on $G_{\delta}$. Then $\phi$ is in $H_{1}^{\infty}\left(G_{\delta}\right)$ if and only if $\phi$ has a free $\delta$-realization.

The third is a Montel theorem.

Theorem 2.4. Let $\left(\phi_{i}\right)_{i=1}^{\infty}$ be a sequence in $H_{1}^{\infty}(U)$. Then there is a subsequence $\left(\phi_{i_{j}}\right)_{j=1}^{\infty}$ and a function $\phi$ in $H_{1}^{\infty}(U)$ such that $\left(\phi_{i_{j}}\right)_{j=1}^{\infty}$ converges to $\phi$ locally uniformly on $U$ in the disjoint union topology.

\section{Proof of Theorem 1.3}

Let $E=V_{\Lambda} \cap G_{\delta}$, and let

$$
E^{[2]}=\left\{(x, y): x, y \in V_{\Lambda} \cap G_{\delta} \cap \mathbb{M}_{m}^{d} \text {, for some } m\right\} .
$$

Let us start with some lemmata. 
Lemma 3.1. Let $\Lambda, x \in \mathbb{M}^{[d]}$. The following are equivalent:

(i) $x \in V_{\Lambda}$.

(ii) There is a homomorphism $\alpha: \mathbb{M}_{\Lambda} \rightarrow \mathbb{M}_{x}$ such that $\alpha\left(\Lambda^{r}\right)=x^{r}$ for $r=1, \ldots, d$.

(iii) The map $p(\Lambda) \mapsto p(x)$ is a well-defined map from $\mathbb{M}_{\Lambda}$ to $\mathbb{M}_{x}$.

(iv) The map $p(\Lambda) \mapsto p(x)$ is a completely bounded homomorphism.

Proof: The equivalence of (i) - (iii) is by definition. That (iii) is equivalent to (iv) is because every bounded homomorphism defined on a finite dimensional space is automatically completely bounded [18].

Lemma 3.2. Let $\phi$ be in $H^{\infty}\left(G_{\delta}\right)$. Then there exists a polynomial $p_{0} \in \mathbb{P}^{d}$ so that

$$
\phi(x)=p_{0}(x) \quad \forall x \in V_{\Lambda} \cap G_{\delta} .
$$

ProOF: By Theorem 2.1, the free function $\phi$ can be uniformly approximated on a free neighborhood of $\Lambda$ by free polynomials. In particular, since $\mathbb{M}_{\Lambda}$ is closed, there is a polynomial $p_{0}$ such that $\phi(\Lambda)=p_{0}(\Lambda)$.

Fix $x \in V_{\Lambda} \cap G_{\delta}$. By another application of the same theorem, there is a free polynomial $p_{1}$ such that $\phi(\Lambda \oplus x)=p_{1}(\Lambda \oplus x)$. Therefore $p_{0}(\Lambda)=p_{1}(\Lambda)$, so by the definition of $V_{\Lambda}$, we also have $p_{0}(x)=p_{1}(x)$. Therefore (3.3) holds, as desired.

We let $\mathcal{V}$ denote the vector space of nc-polynomials on $E$, where we identify polynomials that agree on $E$; and we let $\mathcal{V}_{\mathcal{L}(\mathcal{H}, \mathcal{M})}$ denote the vector space of $\mathcal{L}(\mathcal{H}, \mathcal{M})$-valued nc-polynomials on $E$. As any such polynomial on $E$ is uniquely determined by its values on $\Lambda$, the space of such functions is finite dimensional, if $\mathcal{H}$ and $\mathcal{M}$ are finite dimensional.

Consider the following vector spaces of functions on $E^{[2]}$, where all sums are over a finite set of indices:

$$
\begin{aligned}
& H(E)=\left\{h(y, x)=\sum g_{i}(y)^{*} f_{i}(x): f_{i}, g_{i} \in \mathbb{P}^{d}\right\} \\
& R(E)=\left\{h \in H(E): h(x, y)=h(y, x)^{*}\right\} \\
& C(E)=\left\{h(y, x)=\sum u_{i}(y)^{*}\left[\text { id }-\delta(y)^{*} \delta(x)\right] u_{i}(x):\right. \\
&\left.u_{i} \text { is } \mathcal{L}\left(\mathbb{C}, \mathbb{C}^{J}\right)-\text { valued nc polynomial }\right\} \\
& P(E)=\left\{h(y, x)=\sum f_{i}(y)^{*} f_{i}(x): f_{i} \in \mathbb{P}^{d}\right\}
\end{aligned}
$$


We topologize $H(E)$ with the norm

$$
\|h(y, x)\|=\|h(\Lambda, \Lambda)\|
$$

Lemma 3.4. Let $\mathcal{H}, \mathcal{M}$ be finite dimensional Hilbert spaces, and let $F(y, x)$ be an arbitrary graded $\mathcal{L}(\mathcal{M})$-valued function on $E^{[2]}$. Let $N_{0}=\operatorname{dim}\left(\mathcal{V}_{\mathcal{L}(\mathcal{H}, \mathcal{M})}\right)$. Then if $G$ can be represented in the form

$$
G(y, x)=\sum_{i=1}^{m} g_{i}(y)^{*} F(y, x) g_{i}(x), \quad(x, y) \in E^{[2]}
$$

where $m \in \mathbb{N}$ and $g_{i} \in \mathcal{V}_{\mathcal{L}(\mathcal{H}, \mathcal{M})}$ for $i=1, \ldots, m$, then $G$ can be represented in the form

$$
G(y, x)=\sum_{i=1}^{N_{0}} f_{i}(y)^{*} F(y, x) f_{i}(x), \quad(x, y) \in E^{[2]}
$$

where $f_{i} \in \mathcal{V}_{\mathcal{L}(\mathcal{H}, \mathcal{M})}$ for $i=1, \ldots, N_{0}$.

Proof: Let $\left\langle e_{l}(x)\right\rangle_{l=1}^{N_{0}}$ be a basis of $\mathcal{V}_{\mathcal{L}(\mathcal{H}, \mathcal{M})}$. For each $i=1, \ldots, m$, let

$$
g_{i}(x)=\sum_{l=1}^{N_{0}} c_{i l} e_{l}(x)
$$

Form the $m \times N_{0}$ matrix $C=\left[c_{i l}\right]$. As $C^{*} C$ is an $N_{0} \times N_{0}$ positive semidefinite matrix, there exists an $N_{0} \times N_{0}$ matrix $A=\left[a_{k l}\right]$ such that $C^{*} C=A^{*} A$. This leads to the formula,

$$
\sum_{i=1}^{m} \bar{c}_{i l_{1}} c_{i l_{2}}=\sum_{k=1}^{N_{0}} \bar{a}_{k l_{1}} a_{k l_{2}}
$$


valid for all $l_{1}, l_{2}=1, \ldots, N_{0}$. If $(x, y) \in E^{[2]}$, then

$$
\begin{aligned}
G(y, x) & =\sum_{i=1}^{m} g_{i}(y)^{*} F(y, x) g_{i}(x) \\
& =\sum_{i=1}^{m}\left(\sum_{l=1}^{N_{0}} c_{i l} e_{l}(y)\right)^{*} F(y, x)\left(\sum_{l=1}^{N_{0}} c_{i l} e_{l}(x)\right) \\
& =\sum_{l_{1}, l_{2}=1}^{N_{0}}\left(\sum_{i=1}^{m} \bar{c}_{i l_{1}} c_{i l_{2}}\right) e_{l_{1}}(y)^{*} F(y, x) e_{l_{2}}(x) \\
& =\sum_{l_{1}, l_{2}=1}^{N_{0}}\left(\sum_{k=1}^{N_{0}} \bar{a}_{k l_{1}} a_{k l_{2}}\right) e_{l_{1}}(y)^{*} F(y, x) e_{l_{2}}(x) \\
& =\sum_{k=1}^{N_{0}}\left(\sum_{l=1}^{N_{0}} a_{k l} e_{l}(y)\right)^{*} F(y, x)\left(\sum_{l=1}^{N_{0}} a_{k l} e_{l}(x)\right) .
\end{aligned}
$$

This proves that (3.5) holds with $f_{i}=\sum_{l=1}^{N_{0}} a_{i l} e_{l}$.

Lemma 3.6. $C(E)$ is closed.

Proof: By Lemma 3.4, every element in $C(E)$ can be represented in the form

$$
\sum_{i=1}^{N_{0}} u_{i}(y)^{*}\left[\mathrm{id}-\delta(y)^{*} \delta(x)\right] u_{i}(x)
$$

where $N_{0}=\operatorname{dim} \mathcal{V}_{\mathcal{L}\left(\mathbb{C}, \mathbb{C}^{J}\right)}$. Suppose a sequence of elements of the form (3.7) approaches some $h$ in $H(E)$ at the point $(\Lambda, \Lambda)$ :

$$
\sum_{i=1}^{N_{0}} u_{i}^{(k)}(\Lambda)^{*}\left[\mathrm{id}-\delta(\Lambda)^{*} \delta(\Lambda)\right] u_{i}^{(k)}(\Lambda) \rightarrow h(\Lambda, \Lambda) \text { as } k \rightarrow \infty .
$$

Since $\Lambda \in G_{\delta}$, there is a constant $M$ such that, for each $i$ and $k$,

$$
\left\|u_{i}^{(k)}(\Lambda)\right\| \leq M
$$

Passing to a subsequence, one can assume that each $u_{i}^{(k)}(\Lambda)$ converges to some $u_{i}(\Lambda)$ (since $u_{i}^{(k)}$ is a graded $\mathcal{L}\left(\mathbb{C}, \mathbb{C}^{J}\right)$ valued function and $\left.J<\infty\right)$. By Lemma 3.1, we have

$$
u_{i}^{(k)}(x) \rightarrow u_{i}(x) \quad \forall x \in E .
$$


Therefore, for all $(x, y) \in E^{[2]}$, we have

$$
\begin{aligned}
\sum_{i=1}^{N_{0}} u_{i}^{(k)}(y)^{*}\left[\mathrm{id}-\delta(y)^{*} \delta(x)\right] u_{i}^{(k)}(x) & \rightarrow \sum_{i=1}^{N_{0}} u_{i}(y)^{*}\left[\mathrm{id}-\delta(y)^{*} \delta(x)\right] u_{i}(x) \\
& =h(y, x) .
\end{aligned}
$$

Lemma 3.8. We have $P(E) \subseteq C(E)$.

Proof: We have

$f(y)^{*} f(x)-\sum_{k=0}^{m-1} f(y)^{*} \delta(y)^{k *}[\mathrm{id}-\delta(y) \delta(x)] \delta(x)^{k} f(x)=f(y)^{*} \delta(y)^{m} \delta(x)^{m} f(x)$.

As $m \rightarrow \infty$, the right-hand side of (3.9) goes to zero for every $(x, y) \in E^{[2]}$. Since $C(E)$ is closed by Lemma 3.6, this proves that $f(y)^{*} f(x) \in C(E)$, and hence so are finite sums of this form.

Lemma 3.10. Suppose $\sup \left\{\left\|p_{0}(x)\right\|: x \in E\right\} \leq 1$. Then the function

$$
h(y, x)=\mathrm{id}-p_{0}(y)^{*} p_{0}(x)
$$

is in $C(E)$.

Proof: This will follow from the Hahn-Banach theorem [23, Thm. 3.3.4] if we can can show that $L(h(y, x)) \geq 0$ whenever

$$
L \in R(E)^{*} \quad \text { and } \quad L(g) \geq 0 \forall g \in C(E) .
$$

Assume (3.11) holds, and define $L^{\sharp} \in H(E)^{*}$ by the formula

$$
L^{\sharp}(h(y, x))=L\left(\frac{h(y, x)+h(x, y)^{*}}{2}\right)+i L\left(\frac{h(y, x)-h(x, y)^{*}}{2 i}\right),
$$

and then define sesquilinear forms on $\mathcal{V}$ and $\mathcal{V}_{\mathcal{L}\left(\mathbb{C}, \mathbb{C}^{J}\right)}$ by the formulas

$$
\begin{aligned}
\langle f, g\rangle_{L_{1}} & =L^{\sharp}\left(g(y)^{*} f(x)\right), \quad f, g \in \mathcal{V} \\
\langle F, G\rangle_{L_{2}} & =L^{\sharp}\left(G(y)^{*} F(x)\right), \quad F, G \in \mathcal{V}_{\mathcal{L}\left(\mathbb{C}, \mathbb{C}^{J}\right)} .
\end{aligned}
$$

Observe that Lemma 3.8 implies that $f(y)^{*} f(x) \in C(E)$ whenever $f \in \mathcal{V}$ or $\mathcal{V}_{\mathcal{L}\left(\mathbb{C}, \mathbb{C}^{J}\right)}$. Hence, (3.11) implies that $\langle f, f\rangle_{L_{1}} \geq 0$ for all $f \in \mathcal{V}$, and 
$\langle F, F\rangle_{L_{2}} \geq 0$ for all $F \in \mathcal{V}_{\mathcal{L}\left(\mathbb{C}, \mathbb{C}^{J}\right)}$, i.e., $\langle\cdot, \cdot\rangle_{L_{1}}$ and $\langle\cdot, \cdot\rangle_{L_{2}}$ are pre-inner products on $\mathcal{V}$ and $\mathcal{V}_{\mathcal{L}\left(\mathbb{C}, \mathbb{C}^{J}\right)}$ respectively.

To make them into inner products, choose $\varepsilon>0$ and define

$$
\begin{aligned}
\langle f, g\rangle_{1} & =L^{\sharp}\left(g(y)^{*} f(x)\right)+\varepsilon \operatorname{tr}\left(g(\Lambda)^{*} f(\Lambda)\right), & & f, g \in \mathcal{V} \\
\langle F, G\rangle_{2} & =L^{\sharp}\left(G(y)^{*} F(x)\right)+\varepsilon \operatorname{tr}\left(G(\Lambda)^{*} F(\Lambda)\right), & & F, G \in \mathcal{V}_{\mathcal{L}\left(\mathbb{C}, \mathbb{C}^{J}\right)}(3)
\end{aligned}
$$

We let $\mathrm{H}_{L_{1}}^{2}$ and $\mathrm{H}_{L_{2}}^{2}$ denote the Hilbert spaces $\mathcal{V}$ and $\mathcal{V}_{\mathcal{L}\left(\mathbb{C}, \mathbb{C}^{J}\right)}$ equipped with the inner products (3.12) and (3.13).

The fact that $L$ is non-negative on $C(E)$ means that

$$
\langle F, F\rangle_{L_{2}} \geq\langle\delta F, \delta F\rangle_{L_{2}}
$$

for all $F$ in $\mathcal{V}_{\mathcal{L}\left(\mathbb{C}, \mathbb{C}^{J}\right)}$. Since $\|\delta(\Lambda)\|<1$, we also have

$$
\operatorname{tr}\left(F(\Lambda)^{*} F(\Lambda)\right)>\operatorname{tr}\left(F(\Lambda)^{*} \delta(\Lambda)^{*} \delta(\Lambda) F(\Lambda)\right),
$$

if $F \neq 0$, and combining (3.14) and (3.15) we get that multiplication by $\delta$ is a strict contraction on $\mathrm{H}_{L_{2}}^{2}$.

Let $M$ denote the $d$-tuple of multiplication by the coordinate functions $x^{r}$ on $\mathrm{H}_{L_{1}}^{2}$. We have just shown that $\|\delta(M)\|<1$, so $M$ is in $G_{\delta}$. As $M$ is also in $V_{\Lambda}$, we have that $M$ is in $E$. Therefore $\left\|p_{0}(M)\right\| \leq 1$, by hypothesis. Therefore

$$
\text { id }-p_{0}(M)^{*} p_{0}(M) \geq 0,
$$

and so for all $f$ in $\mathcal{V}$ we have

$$
\begin{aligned}
& L^{\sharp}\left(f(y)^{*} f(x)\right)+\varepsilon \operatorname{tr}\left(f(\Lambda)^{*} f(\Lambda)\right) \geq \\
& \quad L^{\sharp}\left(f(y)^{*} p_{0}(y)^{*} p_{0}(x) f(x)\right)+\varepsilon \operatorname{tr}\left(f(\Lambda)^{*} p_{0}(\Lambda)^{*} p_{0}(\Lambda) f(\Lambda)\right) .
\end{aligned}
$$

Letting $f$ be the function 1 and letting $\varepsilon \rightarrow 0$, we get

$$
L\left(\mathrm{id}-p_{0}(y)^{*} p_{0}(x)\right) \geq 0,
$$

as desired.

We can now prove the theorem.

Proof of Theorem 1.3: (Necessity). Condition (i) follows from Lemma 3.2. Condition (ii) follows because $p_{0}(x)=f(x)$ for $x \in V_{\Lambda} \cap G_{\delta}$, and $f$ is in the unit ball of $H^{\infty}\left(G_{\delta}\right)$, so $\|f(x)\| \leq 1$ for every $x$ in $G_{\delta}$. 
(Sufficiency). Suppose (i) and (ii) hold. By Lemma 3.10, the function

$$
h(y, x)=\mathrm{id}-p_{0}(y)^{*} p_{0}(x)
$$

is in $C(E)$. By Lemma 3.4, there is some positive integer $N \leq \operatorname{dim}\left(\mathcal{V}_{\mathcal{L}\left(\mathbb{C}^{n}, \mathbb{C}^{n}\right)}\right)$ and an $\mathcal{L}\left(\mathbb{C}, \mathbb{C}^{J N}\right)$-valued nc polynomial $u$ such that, for $x, y \in E \cap \mathbb{M}_{n}^{d}$,

$$
\begin{aligned}
h(y, x) & =\operatorname{id}_{\mathbb{C}^{n}}-p_{0}(y)^{*} p_{0}(x) \\
& =u(y)^{*}\left[\operatorname{id}_{\mathbb{C}^{n J N}}-\left(\delta(y)^{*} \otimes \operatorname{id}_{\mathbb{C}^{N}}\right)\left(\delta(x) \otimes \operatorname{id}_{\mathbb{C}^{N}}\right)\right] u(x) .
\end{aligned}
$$

Replace $x$ in (3.16) with $s x s^{-1}$ where $s$ is invertible in $\mathbb{M}_{n}$ and $s x s^{-1}$ is in $G_{\delta}$ to get

$$
\begin{aligned}
& s-p_{0}(y)^{*} s p_{0}(x)= \\
& \quad u(y)^{*}\left[s \otimes \operatorname{id}_{\mathbb{C}^{J N}}-\left(\delta(y)^{*} \otimes \operatorname{id}_{\mathbb{C}^{N}}\right) s \otimes \operatorname{id}_{\mathbb{C}^{J N}}\left(\delta(x) \otimes \operatorname{id}_{\mathbb{C}^{N}}\right)\right] u(x) .
\end{aligned}
$$

Equation (3.17) is true for all $s$ in a neighborhood of the identity, and as linear combinations of such elements span $\mathbb{M}_{n}$, we get that (3.17) actually holds for all $s$ in $\mathbb{M}_{n}$. For $k=1, \ldots, n$, define $\pi_{k}: \mathbb{C}^{n} \rightarrow \mathbb{C}$ by the formula

$$
\pi_{k}(v)=v_{k}, \quad v=\left(v_{1}, \ldots, v_{n}\right) \in \mathbb{C}^{n} .
$$

Letting $s=\pi_{l}^{*} \pi_{k}$ in (3.17) and applying to $v$ and taking the inner product with $w$, where $v$ and $w$ are in $\mathbb{C}^{n}$, leads to

$$
\begin{aligned}
& \left\langle\left[\pi_{l}^{*} \pi_{k}-p_{0}(y)^{*} \pi_{l}^{*} \pi_{k} p_{0}(x)\right] v, w\right\rangle= \\
& \quad\left\langle\left[\pi_{l}^{*} \pi_{k} \otimes \mathrm{id}-\left(\delta(y)^{*} \otimes \mathrm{id}\right)\left(\pi_{l}^{*} \pi_{k} \otimes \mathrm{id}\right)(\delta(x) \otimes \mathrm{id})\right] u(x) v, u(y) w\right\rangle .
\end{aligned}
$$

For each $v \in \mathbb{C}^{n}$ define vectors $p_{v}$ and $q_{v}$ in $\mathbb{C}^{n(1+N J)}$ by

$$
\begin{aligned}
p_{v} & =\left[\begin{array}{c}
\operatorname{id}_{\mathbb{C}^{n}} \\
{\left[\delta(\Lambda) \otimes \mathrm{id}_{\mathbb{C}^{N}}\right] u(\Lambda)}
\end{array}\right] v \\
q_{v} & =\left[\begin{array}{c}
p_{0}(\Lambda) \\
u(\Lambda)
\end{array}\right] v .
\end{aligned}
$$

For each $1 \leq k \leq n$, define vectors $p_{k, v}$ and $q_{k, v}$ in $\mathbb{C}^{1+N J}$ by

$$
\begin{aligned}
p_{k, v} & =\left[\pi_{k} \otimes \mathrm{id}_{\mathbb{C}^{1+N J}}\right] p_{v} \\
q_{k, v} & =\left[\pi_{k} \otimes \mathrm{id}_{\mathbb{C}^{1+N J}}\right] q_{v} .
\end{aligned}
$$


Then (3.18), with $\Lambda$ in place of both $x$ and $y$, becomes

$$
\left\langle p_{k, v}, p_{l, w}\right\rangle=\left\langle q_{k, v}, q_{l, w}\right\rangle \quad \forall v, w \in \mathbb{C}^{n}, \forall 1 \leq k, l \leq n .
$$

So by (3.19), there is an isometry $V$ that maps each $p_{k, v}$ to $q_{k, v}$. If the span of the vectors $\left\{p_{k, v}\right\}$ is not all of $\mathbb{C}^{1+N J}$, we can extend $V$ to the orthocomplement so that it becomes an isometry (indeed, a unitary) from all of $\mathbb{C}^{1+N J}$ to $\mathbb{C}^{1+N J}$.

With respect to the decomposition $\mathbb{C} \oplus \mathbb{C}^{J N}$, write

$$
V=\left[\begin{array}{ll}
A & B \\
C & D
\end{array}\right]
$$

We have

$$
\left[\begin{array}{ll}
A & B \\
C & D
\end{array}\right]\left[\pi_{k} \otimes \operatorname{id}_{\mathbb{C}^{1+N J}}\right]\left[\begin{array}{c}
\operatorname{id}_{\mathbb{C}^{n}} \\
{\left[\delta(\Lambda) \otimes \operatorname{id}_{\mathbb{C}^{N}}\right] u(\Lambda)}
\end{array}\right]=\left[\pi_{k} \otimes \operatorname{id}_{\mathbb{C}^{1+N J}}\right]\left[\begin{array}{c}
p_{0}(\Lambda) \\
u(\Lambda)
\end{array}\right] .
$$

Since (3.20) holds for each $k$, we get that

$$
\left[\begin{array}{ll}
\operatorname{id}_{\mathbb{C}^{n}} \otimes A & \operatorname{id}_{\mathbb{C}^{n}} \otimes B \\
\operatorname{id}_{\mathbb{C}^{n}} \otimes C & \operatorname{id}_{\mathbb{C}^{n}} \otimes D
\end{array}\right]\left[\begin{array}{c}
\operatorname{id}_{\mathbb{C}^{n}} \\
{\left[\delta(\Lambda) \otimes \operatorname{id}_{\mathbb{C}^{n}}\right] u(\Lambda)}
\end{array}\right]=\left[\begin{array}{c}
p_{0}(\Lambda) \\
u(\Lambda)
\end{array}\right]
$$

For $x$ in $G_{\delta} \cap \mathbb{M}_{n}^{d}$, define

$$
\begin{aligned}
& \phi(x)=\operatorname{id}_{\mathbb{C}^{n}} \otimes A+\left[\left(\operatorname{id}_{\mathbb{C}^{n}} \otimes B\right)\left(\delta(x) \otimes \operatorname{id}_{\mathbb{C}^{N}}\right)\right] \\
& \quad\left[\operatorname{id}_{\mathbb{C}^{n}} \otimes \operatorname{id}_{\mathbb{C}^{J N}}-\left(\operatorname{id}_{\mathbb{C}^{n}} \otimes D\right)\left(\delta(x) \otimes \operatorname{id}_{\mathbb{C}^{N}}\right)\right]^{-1} \operatorname{id}_{\mathbb{C}^{n}} \otimes C .
\end{aligned}
$$

Then $\phi$ is in the unit ball of $H^{\infty}\left(G_{\delta}\right)$ by Theorem 2.3. Moreover, by(3.21),

$$
\phi(\Lambda)=p_{0}(\Lambda),
$$

as desired.

\section{The Nevanlinna Problem}

There are two sources of non-uniqueness in the solution of the Pick interpolation problem. The first is the choice of $u$ in (3.16); the second is in the extension of $V$. This problem has been analyzed in the scalar case by J. Ball, T. Trent and V. Vinnikov [6]; their ideas extend to our situation. 
Let us suppose throughout this section that

$$
\Lambda \mapsto p_{0}(\Lambda)
$$

is a solvable Pick problem, and we have found a finite-dimensional space $\mathcal{L}$, an $\mathcal{L}\left(\mathbb{C}, \mathbb{C}^{J} \otimes \mathcal{L}\right)$-valued nc polynomial $u$ satisfying

$$
\operatorname{id}_{\mathbb{C}^{n}}-p_{0}(\Lambda)^{*} p_{0}(\Lambda)=u(\Lambda)^{*}\left[\operatorname{id}_{\mathbb{C}^{n J} \otimes \mathcal{L}}-\left(\delta(\Lambda)^{*} \delta(\Lambda) \otimes \operatorname{id}_{\mathcal{L}}\right)\right] u(\Lambda),
$$

and $V$ satisfying (3.20):

$$
V\left[\pi_{k} \otimes \operatorname{id}_{\mathbb{C} \oplus \mathbb{C}^{J} \otimes \mathcal{L}}\right]\left[\begin{array}{c}
\operatorname{id}_{\mathbb{C}^{n}} \\
{\left[\delta(\Lambda) \otimes \operatorname{id}_{\mathcal{L}}\right] u(\Lambda)}
\end{array}\right]=\left[\pi_{k} \otimes \operatorname{id}_{\left.{\mathbb{C} \oplus \mathbb{C}^{J} \otimes \mathcal{L}}\right]}\left[\begin{array}{c}
p_{0}(\Lambda) \\
u(\Lambda)
\end{array}\right] .\right.
$$

Let $\mathcal{L}_{0}=\mathbb{C} \oplus \mathbb{C}^{J} \otimes \mathcal{L}$, and

$$
\mathcal{N}_{2}:=\vee_{k=1}^{n} \vee_{v \in \mathbb{C}^{n}}\left[\begin{array}{c}
\pi_{k} v \\
{\left[\left(\pi_{k} \otimes \operatorname{id}_{\mathbb{C}^{J} \otimes \mathcal{L}}\right)\left(\delta(\Lambda) \otimes \operatorname{id}_{\mathbb{C}^{N}}\right)\right] u(\Lambda) v}
\end{array}\right] \subseteq \mathcal{L}_{0} .
$$

Let

$$
\mathcal{N}_{1}:=\vee_{k=1}^{n} \vee_{v \in \mathbb{C}^{n}}\left[\begin{array}{c}
\pi_{k} p_{0}(\Lambda) v \\
\left(\pi_{k} \otimes \operatorname{id}_{\mathbb{C}^{J} \otimes \mathcal{L}}\right) u(\Lambda) v
\end{array}\right] \subseteq \mathcal{L}_{0},
$$

and define $\mathcal{M}_{2}=\mathcal{L}_{0} \ominus \mathcal{N}_{2}$ and $\mathcal{M}_{1}=\mathcal{L}_{0} \ominus \mathcal{N}_{1}$. Then $V$ is an isometry from $\mathcal{N}_{2}$ onto $\mathcal{N}_{1}$. Define a unitary

$$
\begin{aligned}
U: \mathcal{M}_{1} \oplus \mathcal{M}_{2} \oplus \mathcal{N}_{2} & \rightarrow \mathcal{M}_{2} \oplus \mathcal{M}_{1} \oplus \mathcal{N}_{1} \\
{\left[\begin{array}{l}
m_{1} \\
m_{2} \\
n_{2}
\end{array}\right] } & \mapsto\left[\begin{array}{c}
m_{2} \\
m_{1} \\
V n_{2}
\end{array}\right] .
\end{aligned}
$$

By identifying $\mathcal{M}_{1} \oplus \mathcal{M}_{2} \oplus \mathcal{N}_{2} \cong \mathcal{M}_{1} \oplus \mathbb{C} \oplus \mathbb{C}^{J} \otimes \mathcal{L}$ and $\mathcal{M}_{2} \oplus \mathcal{M}_{1} \oplus \mathcal{N}_{1} \cong$ $\mathcal{M}_{2} \oplus \mathbb{C} \oplus \mathbb{C}^{J} \otimes \mathcal{L}$, we can think of $U$ as a unitary from $\mathbb{C} \oplus \mathcal{M}_{1} \oplus \mathbb{C}^{J} \otimes \mathcal{L}$ to $\mathbb{C} \oplus \mathcal{M}_{2} \oplus \mathbb{C}^{J} \otimes \mathcal{L}$, and it has a corresponding transfer function $G$ that is a free $\mathcal{L}\left(\mathbb{C} \oplus \mathcal{M}_{1}, \mathbb{C} \oplus \mathcal{M}_{2}\right)$-valued rational function (since all the spaces are finite dimensional). Write this $G$ as

$$
G=\underset{\mathcal{M}_{2}}{\mathbb{C}}\left(\begin{array}{ll}
G_{11} & G_{12} \\
G_{21} & G_{22}
\end{array}\right) .
$$


Theorem 4.6. The function $\phi$ in ball $\left(H^{\infty}\left(G_{\delta}\right)\right)$ satisfies $\phi(\Lambda)=p_{0}(\Lambda)$ if and only if, for some $u$ satisfying (4.2) and $G$ the transfer function of $U$ in (4.4), there is a function $\Theta$ in $\operatorname{ball}\left(H_{\mathcal{L}\left(\mathcal{M}_{1}, \mathcal{M}_{2}\right)}^{\infty}\left(G_{\delta}\right)\right)$ such that

$$
\phi=G_{11}+G_{12} \Theta\left(I_{\mathcal{M}_{1}}-G_{22} \Theta\right)^{-1} G_{21} .
$$

Proof: $(\Leftarrow)$ This is a straightforward calculation.

$(\Rightarrow)$ By Theorem 2.3, $\phi$ has a free $\delta$-realization, and by Lemma 3.4, we can assume that $\left\{u(x): x \in V_{\Lambda}\right\}$ lie in a finite dimensional space that we can embed in $\mathbb{C}^{J} \otimes \mathcal{L}$. So we can assume that $\phi$ is the transfer function of some unitary $X: \mathbb{C} \oplus \mathbb{C}^{J} \otimes \mathcal{K} \rightarrow \mathbb{C} \oplus \mathbb{C}^{J} \otimes \mathcal{K}$, and that $\mathcal{L} \subseteq \mathcal{K}$. For $x \in G_{\delta} \cap \mathbb{M}_{m}^{d}$ we have

$$
\left[\operatorname{id}_{\mathbb{C}^{m}} \otimes X\right]\left[\begin{array}{c}
\operatorname{id}_{\mathbb{C}^{m}} \\
\left(\delta(x) \otimes \operatorname{id}_{\mathcal{K}}\right) \xi(x)
\end{array}\right]=\left[\begin{array}{l}
\phi(x) \\
\xi(x)
\end{array}\right] .
$$

Let $\mathcal{K}^{\prime}=\mathcal{K} \ominus \mathcal{L}$. Then

$$
\begin{aligned}
& \mathcal{N}_{2} \quad \mathcal{M}_{2} \oplus \mathbb{C}^{J} \otimes \mathcal{K}^{\prime}
\end{aligned}
$$

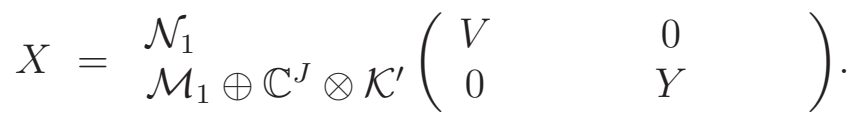

Let $\Theta$ be the transfer function of $Y$. Then we claim that (4.7) holds.

Let $x \in G_{\delta} \cap \mathbb{M}_{m}^{d}$ and $v \in \mathbb{C}^{m}$ be fixed for now. Let

$$
\begin{aligned}
& p=v \oplus\left(\delta(x) \otimes \operatorname{id}_{\mathcal{K}}\right) \xi(x) v=n_{2} \oplus m_{2} \oplus h_{2} \\
& q=\phi(x) v \oplus \xi(x) v \quad=n_{1} \oplus m_{1} \oplus h_{1}
\end{aligned}
$$

where $n_{2} \in \mathbb{C}^{m} \otimes \mathcal{N}_{2}, m_{2} \in \mathbb{C}^{m} \otimes \mathcal{M}_{2}, n_{1} \in \mathbb{C}^{m} \otimes \mathcal{N}_{1}, m_{1} \in \mathbb{C}^{m} \otimes \mathcal{M}_{1}$ and $h_{2}, h_{1} \in \mathbb{C}^{m} \otimes \mathbb{C}^{J} \otimes \mathcal{K}^{\prime}$. Note from (4.8) that

$$
\left[\operatorname{id}_{\mathbb{C}^{m}} \otimes X\right] p=q .
$$

Let $P^{\prime}$ be the projection from $\mathbb{C}^{J} \otimes \mathcal{K}$ to $\mathbb{C}^{J} \otimes \mathcal{K}^{\prime}$. As $\delta(x) \otimes \mathrm{id}_{\mathcal{K}}$ commutes with $\operatorname{id}_{\mathbb{C}^{m}} \otimes P^{\prime}$, we get from (4.10) that

$$
\left[\delta(x) \otimes \operatorname{id}_{\mathcal{K}^{\prime}}\right] h_{1}=h_{2} .
$$

Therefore

$$
\left[\operatorname{id}_{\mathbb{C}^{m}} \otimes Y\right]\left(m_{2} \oplus\left[\delta(x) \otimes \operatorname{id}_{\mathcal{K}^{\prime}}\right] h_{1}\right)=m_{1} \oplus h_{1} .
$$

As $\Theta$ is the transfer function of $Y,(4.12)$ implies that

$$
\Theta(x) m_{2}=m_{1} \text {. }
$$


Let $P$ be the projection from $\mathbb{C}^{m} \otimes \mathbb{C}^{J} \otimes \mathcal{K}$ onto $\mathbb{C}^{m} \otimes \mathbb{C}^{J} \otimes \mathcal{L}$, and let $\eta=P \xi(x) v$. Then under the identifications of $\mathcal{N}_{1} \oplus \mathcal{M}_{1}$ and $\mathcal{N}_{2} \oplus \mathcal{M}_{2}$ with $\mathbb{C} \oplus \mathbb{C}^{J} \otimes \mathcal{L}$, we get

$$
\begin{aligned}
& n_{1} \oplus m_{1}=\phi(x) v \oplus \eta \\
& n_{2} \oplus m_{2}=v \oplus\left(\delta(x) \otimes \operatorname{id}_{\mathcal{L}}\right) \eta .
\end{aligned}
$$

Then from (4.4)

$$
U: v \oplus m_{1} \oplus\left(\delta(x) \otimes \operatorname{id}_{\mathcal{L}}\right) \eta \mapsto \phi(x) v \oplus m_{2} \oplus \eta .
$$

By (4.13) this gives

$$
\left(\begin{array}{ll}
G_{11}(x) & G_{12}(x) \\
G_{21}(x) & G_{22}(x)
\end{array}\right)\left(\begin{array}{c}
v \\
\Theta(x) m_{2}
\end{array}\right)=\left(\begin{array}{c}
\phi(x) v \\
m_{2}
\end{array}\right) .
$$

As (4.14) holds for all choices of $x$ and $v$, we get (4.7), as desired.

\section{$5 \quad$ Extending functions defined on varieties}

Proof of Theorem 1.5: Without loss of generality, assume that

$$
\sup _{x \in \mathfrak{D} \cap G_{\delta}}\|f(x)\|=1 .
$$

Choose a sequence $\left(\lambda_{j}\right)_{j=1}^{\infty}$ in $G_{\delta} \cap \mathfrak{v}$ that is dense in the disjoint union topology, so for all $\varepsilon>0$, for all $x \in G_{\delta} \cap \mathfrak{v}$, there exists some $\lambda_{j}$ such that $\max _{1 \leq r \leq d}\left\|\lambda_{j}^{r}-x^{r}\right\|<\varepsilon$.

Let $\Lambda_{n}=\oplus_{j=1}^{n} \lambda_{j}$. By Theorem 2.1, $f$ is locally approximable by polynomials, and so has the property that

$$
\forall x \in \mathfrak{v} \cap G_{\delta}, f(x) \in \mathbb{M}_{x} .
$$

Therefore there is some polynomial $p_{n} \in \mathbb{P}^{d}$ such that

$$
p_{n}\left(\Lambda_{n}\right)=f\left(\Lambda_{n}\right) .
$$

Moreover, if $x \in \mathfrak{v} \cap G_{\delta}$, then by Theorem 2.1 again, one can approximate $f$ at $x \oplus \Lambda_{n}$ by a sequence of free polynomials, and so by Lemma 3.1

$$
\forall x \in \mathfrak{v} \cap G_{\delta}, f(x)=p_{n}(x) .
$$


As $V_{\Lambda} \subseteq \mathfrak{V}$, putting (5.2), (5.3) and (5.1) together, the hypotheses of Theorem 1.3 are satisfied, so there exists $\phi_{n}$ in $H_{1}^{\infty}\left(G_{\delta}\right)$ such that

$$
\phi_{n}\left(\Lambda_{n}\right)=f\left(\Lambda_{n}\right)
$$

By Theorem 2.4, some subsequence of $\phi_{n}$ converges locally uniformly (in the disjoint union topology) to a function $\phi$ in $H_{1}^{\infty}\left(G_{\delta}\right)$. Moreover, for each $j$, $\phi\left(\lambda_{j}\right)=f\left(\lambda_{j}\right)$, so by continuity, $\phi$ is an extension of $f$.

\section{$6 \quad$ Examples}

Example 6.1 Let $q_{1}, \ldots, q_{m}$ be polynomials in $d$ commuting variables, and let $V=\left\{z \in \mathbb{C}^{d}: q_{i}(z)=0, i=1, \ldots, m\right\}$. Let $f$ be a (scalar-valued) holomorphic function defined on $V \cap \mathbb{D}^{d}$.

Let $T$ be a $d$-tuple of commuting matrices that are strict contractions, and such that $q_{i}(T)=0$ for $i=1, \ldots, m$. If they are simultaneously diagonizable, then their joint eigenvalues lie in $V \cap \mathbb{D}^{d}$, and it makes sense to define $f(T)$ by applying $f$ to the diagonal entries, in the basis of joint eigenvectors. If the matrices are not simultaneously diagonizable, then one can still define $f(T)$, either by the Taylor functional calculus [24], or, more constructively, as in $[2]$.

Let us write $\mathcal{F}$ for the set of all $T=\left(T^{1}, \ldots, T^{d}\right)$ of commuting matrices such that $q_{i}(T)=0, i=1, \ldots, m$, and such that $\|T\|<1$. Note that $\mathcal{F}=$ $\mathfrak{D} \cap G_{\delta}$, where $\mathfrak{A}$ is the algebra generated by $q_{1}, \ldots, q_{m}$ and the polynomials $\left\{x^{i} x^{j}-x^{j} x^{i}: 1 \leq i<j \leq d\right\}, \mathfrak{D}=\operatorname{Var}(\mathfrak{a})$, and $\delta(x)$ is the diagonal matrix with entries $x^{1}, x^{2}, \ldots, x^{d}$. Define a norm on holomorphic functions on $V \cap \mathbb{D}^{d}$ by

$$
\|f\|_{\mathfrak{v}_{\cap G_{\delta}}}=\sup \{\|f(T)\|: T \in \mathcal{F}\} .
$$

To apply Theorem 1.5, we need to know that $f$ is a free holomorphic function on $\mathfrak{D} \cap G_{\delta}$, in other words that locally in $G_{\delta}$ it extends to a free holomorphic function (i.e. it can be applied to non-commuting matrices). This is true, and is proved in [2]. Then Theorem 1.5 asserts that there is a bounded extension $\phi$ of $f$, defined on the set $\left\{R \in \mathbb{M}^{d}:\|R\|<1\right\}$, if and only if $\|f\|_{\mathfrak{v}_{\cap G_{\delta}}}$ is finite. Moreover, if this quantity is finite, then $\phi$ can be found with exactly this norm. In particular, an extension to the non-commuting ball $G_{\delta}$ can always be found with the same norm as is attained by evaluating on commuting matrices in the variety. 
Example 6.2 Specializing the previous example to the case $d=2$, and using Andô's inequality [5], we conclude the following: if we wish to extend a polynomial $p_{0}$ off $V \cap \mathbb{D}^{2}$, where $V$ is the joint sero set of the $q_{i}$ 's, then the minimum norm of the extension $\phi$ is the same when calculated as a scalarvalued function in $H^{\infty}\left(\mathbb{D}^{2}\right)$, as a function on pairs of commuting contractive matrices, or as a function on pairs of contractive matrices. The norm is attained, and is given by

$$
\begin{array}{r}
\sup _{n \in \mathbb{N}} \sup \left\{\left\|p_{0}(T)\right\|: T \in \mathbb{M}_{n}^{2},\left\|T^{1}\right\|<1,\left\|T^{2}\right\|<1,\right. \\
\left.T^{1} T^{2}=T^{2} T^{1}, q_{i}(T)=0 \forall 1 \leq i \leq m\right\} .
\end{array}
$$

Unless $V \cap \mathbb{D}^{2}$ is a retract of $\mathbb{D}^{2}$, one can by [3] always find some $p_{0}$ so that (6.3) is strictly greater than

$$
\sup \left\{\left|p_{0}(z)\right|: z \in \mathbb{D}^{2} \cap V\right\} .
$$

Example 6.4 Suppose $\delta(x)$ has first column $x^{1}, \ldots, x^{d}$ and its other entries zero, so

$$
G_{\delta}=\left\{T: T^{1 *} T^{1}+\cdots T^{d *} T^{d}<1\right\} .
$$

(This is called the row ball). Suppose $\Lambda, H \in G_{\delta} \cap \mathbb{M}_{n}^{d}$ and one wishes to solve the interpolation problem

$$
\begin{aligned}
\phi(\Lambda) & =W \\
D \phi(\Lambda)[H] & =X,
\end{aligned}
$$

where $D \phi(\Lambda)[H]$, the derivative of $\phi$ at $\Lambda$ in the direction $H$, is defined by

$$
D \phi(\Lambda)[H]=\lim _{t \rightarrow 0} \frac{\phi(\Lambda+t H)-\phi(\Lambda)}{t} .
$$

A necessary condition to find a function $\phi \in H^{\infty}\left(G_{\delta}\right)$ solving this problem is that there is some free polynomial $p_{0}$ with $p_{0}(\Lambda)=W$ and $D p_{0}(\Lambda)[H]=X$. The minimum norm of a solution can be found from Theorem 1.5 by letting

$$
\mathfrak{A}=\left\{p \in \mathbb{P}^{d}: p(\Lambda)=0, D p(\Lambda)[H]=0\right\},
$$

$\mathfrak{v}=\operatorname{Var}(\mathfrak{a})$, and calculating

$$
\sup _{x \in \mathfrak{b} \cap G_{\delta}}\left\|p_{0}(x)\right\|
$$


The problem can also be solved using Theorem 1.3, as (6.5) is the same as solving the one point problem

$$
\left(\begin{array}{cc}
\Lambda & H \\
0 & \Lambda
\end{array}\right) \mapsto\left(\begin{array}{cc}
W & X \\
0 & W
\end{array}\right)
$$

since by [12, Prop 2.5], for any continuous nc-function $f$, one has

$$
f\left(\begin{array}{cc}
\Lambda & H \\
0 & \Lambda
\end{array}\right)=\left(\begin{array}{cc}
f(\Lambda) & D f(\Lambda)[H] \\
0 & f(\Lambda)
\end{array}\right)
$$

\section{References}

[1] J. Agler and J.E. McCarthy. Global holomorphic functions in several non-commuting variables. To appear.

[2] J. Agler and J.E. McCarthy. Operator theory and the Oka extension theorem. To appear.

[3] J. Agler and J.E. McCarthy. Norm preserving extensions of holomorphic functions from subvarieties of the bidisk. Ann. of Math., 157(1):289-312, 2003.

[4] D. Alpay and D. S. Kalyuzhnyi-Verbovetzkii. Matrix-J-unitary noncommutative rational formal power series. In The state space method generalizations and applications, volume 161 of Oper. Theory Adv. Appl., pages 49-113. Birkhäuser, Basel, 2006.

[5] T. Andô. On a pair of commutative contractions. Acta Sci. Math. (Szeged), 24:88-90, 1963.

[6] J.A. Ball, T.T. Trent, and V. Vinnikov. Interpolation and commutant lifting for multipliers on reproducing kernel Hilbert spaces. In Operator Theory and Analysis, pages 89-138. Birkhäuser, Basel, 2001.

[7] Joseph A. Ball, Gilbert Groenewald, and Tanit Malakorn. Conservative structured noncommutative multidimensional linear systems. In The state space method generalizations and applications, volume 161 of Oper. Theory Adv. Appl., pages 179-223. Birkhäuser, Basel, 2006. 
[8] H. Cartan. Séminaire Henri Cartan 1951/2. W.A. Benjamin, New York, 1967.

[9] Jakob Cimpric, J. William Helton, Scott McCullough, and Christopher Nelson. Real nullstellensatze and *-ideals in *-algebras. http://arxiv.org/pdf/1302.4722v2.pdf.

[10] J. William Helton. "Positive" noncommutative polynomials are sums of squares. Ann. of Math. (2), 156(2):675-694, 2002.

[11] J. William Helton, Igor Klep, and Scott McCullough. Analytic mappings between noncommutative pencil balls. J. Math. Anal. Appl., 376(2):407$428,2011$.

[12] J. William Helton, Igor Klep, and Scott McCullough. Proper analytic free maps. J. Funct. Anal., 260(5):1476-1490, 2011.

[13] J. William Helton and Scott McCullough. Every convex free basic semialgebraic set has an LMI representation. Ann. of Math. (2), 176(2):9791013, 2012.

[14] J. William Helton and Scott A. McCullough. A Positivstellensatz for non-commutative polynomials. Trans. Amer. Math. Soc., 356(9):37213737 (electronic), 2004.

[15] G.M. Henkin and P.L. Polyakov. Prolongement des fonctions holomorphes bornes d'une sous-varit du polydisque. Comptes Rendus Acad. Sci. Paris Sér. I Math., 298(10):221-224, 1984.

[16] Dmitry S. Kaliuzhnyi-Verbovetskyi and Victor Vinnikov. Foundations of non-commutative function theory. arXiv:1212.6345.

[17] Greg Knese. Polynomials defining distinguished varieties. Trans. Amer. Math. Soc., 362(11):5635-5655, 2010.

[18] V.I. Paulsen. Completely bounded maps and operator algebras. Cambridge University Press, Cambridge, 2002.

[19] Gelu Popescu. Free holomorphic functions on the unit ball of $B(\mathcal{H})^{n}$. J. Funct. Anal., 241(1):268-333, 2006. 
[20] Gelu Popescu. Free holomorphic functions and interpolation. Math. Ann., 342(1):1-30, 2008.

[21] Gelu Popescu. Free holomorphic automorphisms of the unit ball of $B(\mathcal{H})^{n}$. J. Reine Angew. Math., 638:119-168, 2010.

[22] Gelu Popescu. Free biholomorphic classification of noncommutative domains. Int. Math. Res. Not. IMRN, (4):784-850, 2011.

[23] W. Rudin. Functional Analysis. McGraw-Hill, New York, 1991.

[24] J.L. Taylor. The analytic functional calculus for several commuting operators. Acta Math., 125:1-38, 1970.

[25] J.L. Taylor. Functions of several non-commuting variables. Bull. Amer. Math. Soc., 79:1-34, 1973.

[26] Dan Voiculescu. Free analysis questions. I. Duality transform for the coalgebra of $\partial_{X: B}$. Int. Math. Res. Not., (16):793-822, 2004.

[27] Dan-Virgil Voiculescu. Free analysis questions II: the Grassmannian completion and the series expansions at the origin. J. Reine Angew. Math., 645:155-236, 2010. 